\title{
Stabilising a cart inverted pendulum with an augmented PID control scheme
}

\author{
Indrazno Siradjuddin ${ }^{1}$, Zakiyah Amalia ${ }^{1 *}$, Budhy Setiawan ${ }^{1}$, Ferdian Ronilaya $^{1}$, Erfan Rohadi ${ }^{2}$, Awan Setiawan ${ }^{1}$, Cahya \\ Rahmad $^{2}$, and Supriatna Adhisuwignjo ${ }^{1}$
}

${ }^{1}$ Malang State Polytechnic, Electrical Engineering Department, Malang, Indonesia

${ }^{2}$ Malang State Polytechnic, Information Technology Department, Malang, Indonesia

\begin{abstract}
A cart inverted pendulum is an under actuated system that highly unstable and nonlinear. Therefore, it makes a good problem example which attracts control engineers to validate the developed control algorithms. In this paper, an augmented PID control algorithm is proposed to stabilise a cart inverted pendulum at the desired state. The derivation of a mathematical model of the cart inverted pendulum using Lagrange's equation is discussed in detail. The system dynamics is illustrated to understand better the behaviour of the system. A simulation program has been developed to verify the performance of the proposed control algorithm. The system dynamic behaviours with respect to the variation of the controller parameters are analysed and discussed. Controllers parameters are expressed into two PID gain sets which associated with 2 dynamic states: the cart position $(x)$ and the pendulum angle $(\theta)$. It can be concluded from the simulation result that the proposed control algorithm can perform well where acceptable steady errors can be achieved. The best response from the cart inverted pendulum system has been obtained with the value of $k_{P_{x}} 190, k_{D_{x}} 50, k_{I_{x}} 5, k_{P_{\theta}} 140, k_{D_{\theta}} 5$, and $k_{I_{\theta}} 25$.
\end{abstract}

\section{Introduction}

The Cart Inverted Pendulum is an example of a nonlinear [1], unstable [2] and under actuated system [3]. The nonlinear system means the system has many possible responses although the same input is given. A normal pendulum or a pendulum that facing downwards is a stable system, but an inverted pendulum is an unstable system. In the cart-inverted pendulum, the output variables are expressed by its position of the cart and the rod angle, and a horizontal force [4] expresses the input variable. The cart-inverted pendulum is a system that usually used for testing many control algorithms [5]. There are some control algorithms that can be used for stabilising a cart-inverted pendulum such as Linear Quadratic Regulator (LQR) [6], neural network [7], genetic algorithm [8], fuzzy control [9], and PID [10] which have been studied by many researchers. The studies about the stability of a cart-inverted pendulum is very useful for developing real world application systems, for instances: a segway, an earthquake resistant building design, a human walking, etc.

An augmented PID control scheme is proposed for stabilising a cart inverted pendulum in this work. The system modelling is discussed in Section 2. In this section, the mathematical model for cart and pendulum is derived by Lagrange Equation. The open and closed loop control are discussed in Section 3. The modelling of cart inverted pendulum with PID is derived in Section 4.
The simulation results are shown in Section 5. Finally, Section 6 presents the conclusion of the work.

\section{System modelling}

\subsection{Lagrange's equation}

A complex system dynamic can be described in efficient way using Lagrange's equation. The complicated vector analysis that needed for describing forces applied on a mechanical system can be reduced by Langrange's equation.

A set of generalised coordinate $\boldsymbol{q}=$ $\left\{q_{1}, \ldots, q_{i}, \ldots, q_{n}\right\}$ is a representation of the fundamental principle of Lagrange's equation, where $q_{i}$ is an independent degree of freedom of the system which combines the constraints unique to the system, i.e., the communication between parts of the system. The total generalised coordinates is do noted by $n$.

The Lagrange's equation is expressed by the system's kinetic $\boldsymbol{K}$ and potential energy $\boldsymbol{P}$ which described as follows

$$
\mathcal{L}=K(\boldsymbol{q}, \dot{q})-P(\mathbf{q})
$$

Where the function of kinetic energy in terms of the generalised coordinate $\boldsymbol{q}$ and its derivative $\dot{\boldsymbol{q}}$. The function of potential energy is described in terms of only the generalised coordinate $\boldsymbol{q}$.

\footnotetext{
*Corresponding author: zakiyah_amalia@polinema.ac.id
} 
The equations of desired motion are derived using

$$
\frac{d}{d t}\left(\frac{\partial \mathcal{L}}{\partial \dot{q}_{l}}\right)-\frac{\partial \mathcal{L}}{\partial q_{i}}=Q_{i}
$$

Where $Q_{i}$ shows the external force that applied in term of $q_{i}$ coordinate. The development of the Lagrange's equation of the cart-inverted pendulum is presented in the following section.

\subsection{Lagrange's equation of the system}

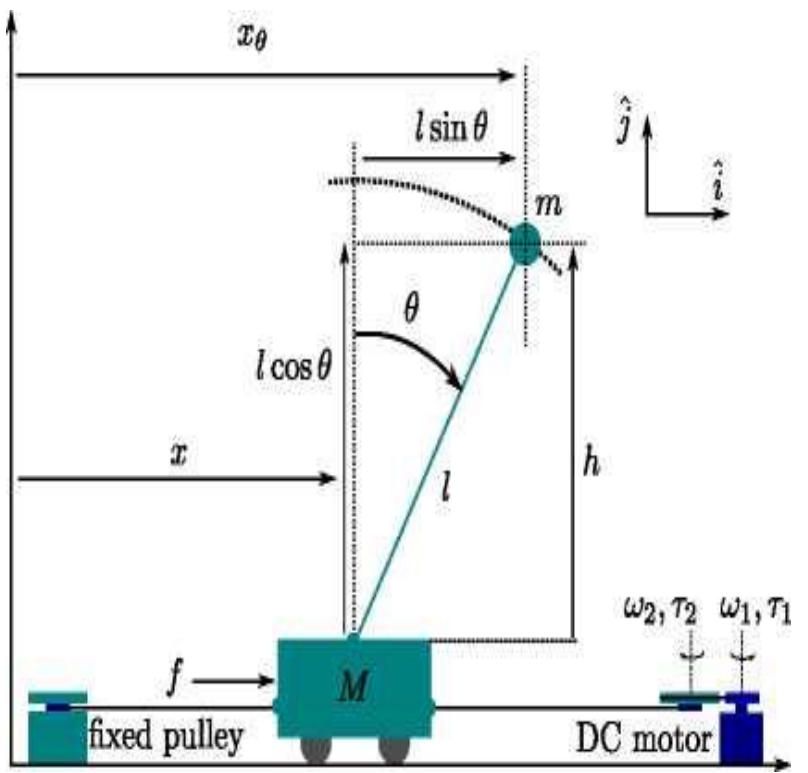

Fig. 1. The inverted pendulum on a cart system.

In Fig. 1 , the motion of the cart is only in $\hat{\imath}$ direction, thus the total kinetic energy of the cart can be expressed as

$$
K_{M}=\frac{1}{2} M \dot{x}^{2}
$$

The motion of pendulum are expressed in $\hat{\jmath}$ and $\hat{\imath}$ directions, hence, the total kinetic energy can be formulated as

$$
K_{m}=\frac{1}{2} m\left(\dot{x}_{\theta}^{2}+\dot{h}^{2}\right)
$$

Fig. 1 shows that

$$
\begin{gathered}
x_{\theta}=x+l \sin \theta \\
h=l \cos \theta
\end{gathered}
$$

Where $x$ is the position of the cart, $x_{\theta}$ is the projected pendulum position on the horizontal axis, and $h$ is the projected pendulum position on the vertical axis. The first derivatives of Eq. (5) and Eq. (6), are determined as

$$
\begin{gathered}
\dot{x_{\theta}}=\dot{x}+l \dot{\theta} \cos \theta \\
\dot{h}=-l \dot{\theta} \sin \theta
\end{gathered}
$$

Hence, the kinetic energy of pendulum in Eq. (4) can be rewritten as

$$
\begin{gathered}
K_{m}=\frac{1}{2} m\left((\dot{x}+l \dot{\theta} \cos \theta)^{2}+(-l \dot{\theta} \sin \theta)^{2}\right) \\
K_{m}=\frac{1}{2} m\left(\dot{x}^{2}+2 \dot{x} l \dot{\theta} \cos \theta+l^{2} \dot{\theta}^{2}\right)
\end{gathered}
$$

Combining the kinetic energy formulations in Eq. (10) and Eq. (3), one can verify that the total kinetic energy of the system can be expressed as

$$
K=K_{M}+K_{m}
$$

$$
K=\frac{1}{2} M \dot{x}^{2}+\frac{1}{2} m\left(\dot{x}^{2}+2 \dot{x} l \dot{\theta} \cos \theta+l^{2} \dot{\theta}^{2}\right)
$$

The pendulum mass $m$ is only the potential energy involved in the system

$$
\begin{gathered}
P=m g h \\
P=m g l \cos \theta
\end{gathered}
$$

Then, the Lagrangian equation Eq. (15) can be fully determined using Eq. (12) and Eq. (14), as follows

$$
\mathcal{L}=\frac{1}{2} M \dot{x}^{2}+\frac{1}{2} m\left(\dot{x}^{2}+2 \dot{x} l \dot{\theta} \cos \theta+l^{2} \dot{\theta}^{2}\right)-m g l \cos \theta
$$

The angle of the pendulum with respect to the $\widehat{J}$ direction and the displacement of the cart in the $\hat{\imath}$ direction with respect to the origin specifically defined the motion of the inverted pendulum on a cart.

Therefore, the system only has two degrees of freedom represented by $\theta$ and $x$, the system dynamics must be expressed in terms of $\theta$ and $x$. Thus, $\theta$ and $x$ can be selected as the elements of the generalised coordinate vector $\boldsymbol{q}$. The Lagrange's equation Eq. (2) can be expressed using this selection for each generalised coordinate:

$$
\begin{aligned}
& \frac{d}{d t}\left(\frac{\partial \mathcal{L}}{\partial \dot{x}}\right)-\frac{\partial \mathcal{L}}{\partial x}=f \\
& \frac{d}{d t}\left(\frac{\partial \mathcal{L}}{\partial \dot{\theta}}\right)-\frac{\partial \mathcal{L}}{\partial \theta}=0
\end{aligned}
$$

The external forces that applied to the system is assumed that there is no external torque applied to the pendulum and the external force only applied on a cart in $\hat{\imath}$ direction. Deriving for each term in the differential equation in Eq. (16) and Eq. (17), we have

$$
\left(\frac{\partial \mathcal{L}}{\partial \dot{x}}\right)=(M+m) \dot{x}+m l \dot{\theta} \cos \theta
$$

$$
\frac{d}{d t}\left(\frac{\partial \mathcal{L}}{\partial \dot{x}}\right)=(M+m) \ddot{x}+m l \ddot{\theta} \cos \theta-m l \dot{\theta}^{2} \sin \theta
$$




$$
\frac{\partial \mathcal{L}}{\partial x}=0
$$

and

$$
\begin{gathered}
\left(\frac{\partial \mathcal{L}}{\partial \dot{\theta}}\right)=m l \dot{x} \cos \theta+m l^{2} \dot{\theta} \\
\frac{d}{d t}\left(\frac{\partial \mathcal{L}}{\partial \dot{\theta}}\right)=m l(\ddot{x} \cos \theta-\dot{\theta} \dot{x} \sin \theta)+m l^{2} \ddot{\theta} \\
\frac{\partial \mathcal{L}}{\partial \theta}=-m \dot{x} l \dot{\theta} \sin \theta+m g l \sin \theta
\end{gathered}
$$

Hence, the Lagrange's equation for the cart and the pendulum can be formulated as

$$
\begin{aligned}
f= & (M+m) \ddot{x}+m l \ddot{\theta} \cos \theta-m l \dot{\theta}^{2} \sin \theta \\
0= & m l(\ddot{x} \cos \theta-\dot{\theta} \dot{x} \sin \theta)+m l^{2} \ddot{\theta} \\
& -(-m \dot{x} l \dot{\theta} \sin \theta+m g l \sin \theta)
\end{aligned}
$$

It can be seen that Eq. (24) and Eq. (25) have terms $\sin ()$ and $\cos ()$. Hence, terms $\dot{\theta}^{2}$ and $\dot{\theta} \theta$ can be neglected. Stabilising the pendulum vertically with small deflection of $\theta$ is the aim of the controller that will be designed. It is assumed that the pendulum will be initialised near to the reference angle (upright), thus it can be approximated that $\sin \theta \approx \theta$ and $\cos \theta \approx 1$. Using assumptions that mentioned before, the linearised Lagrange's equation can be derived as follows

$$
\begin{gathered}
f=(M+m) \ddot{x}+m l \ddot{\theta} \\
0=\ddot{x}+l \ddot{\theta}-g \theta
\end{gathered}
$$

\section{Open and closed loop control}

In a cart inverted pendulum system, the motion dynamic of the cart can be controlled using an external force or torque. The motions dynamic state is measured with position, velocity, and possibly acceleration sensors. In the case of a cart inverted pendulum system, two motions dynamic states are measured: the motion of the cart and the motion of the pendulum. These two motion states are coupled. In order, to stabilise the pendulum at the equilibrium position, the desired external force applied to the system should be computed. We denoted by $Q$. The position, the velocity and the acceleration of the system are denoted by $q, \dot{q}, \ddot{q}$, respectively. The motion dynamics is then described by:

$$
Q_{c}=D\left(\boldsymbol{q}_{\boldsymbol{d}}\right) \ddot{\boldsymbol{q}}_{\boldsymbol{d}}+H\left(\boldsymbol{q}_{\boldsymbol{d}}, \boldsymbol{q}_{\boldsymbol{d}}\right)+\boldsymbol{G}\left(\boldsymbol{q}_{\boldsymbol{d}}\right)
$$

Where the subscripts $\mathrm{c}$ in $Q_{c}$ means a controlled force and $\mathrm{d}$ in $\boldsymbol{q}_{\boldsymbol{d}}$ expresses the desired states. The desired position $\boldsymbol{q}_{\boldsymbol{d}}$ can be reached by giving the external force $Q_{c}$. The external force that described in Eq.(28) is an open loop control algorithm. The block diagram of open loop control is shown in Fig.2

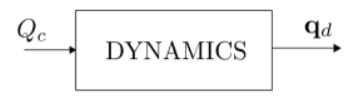

Fig. 2. Illustration of open-loop control algorithm.

In the open loop system, there is no mechanism to compensate any possible error. A feedback control algorithm is needed to resolve this problem.

Linear control technique is a simple technique for controlling robots. Linear control technique is a method based on linearization of the system model equations with a combination of proportional, integral, derivative gain, or any combination of them.

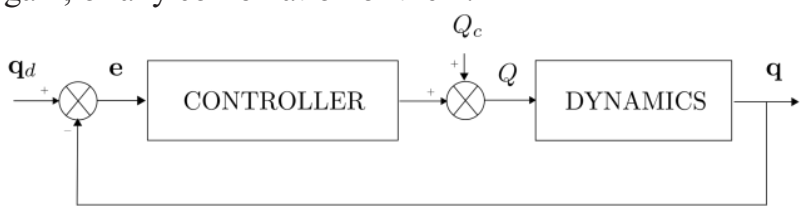

Fig. 3. Illustration of feedback control algorithm.

Fig. 3 illustrate the feedback control algoritm with a new motion dynamics that described by:

$$
Q=Q_{c}+\boldsymbol{k}_{D} \dot{\boldsymbol{e}}+\boldsymbol{k}_{P} \boldsymbol{e}+\boldsymbol{k}_{\boldsymbol{I}} \int_{0}^{t} \boldsymbol{e} d t
$$

Where proportional gain control $\boldsymbol{k}_{P}$, derivative gain control $\boldsymbol{k}_{\boldsymbol{D}}$, and integral gain control $\boldsymbol{k}_{\boldsymbol{I}}$ added to the system. The feedback error or the difference between actual and desired value is denoted by $\boldsymbol{e}$, where:

$$
\boldsymbol{e}=\boldsymbol{q}-\boldsymbol{q}_{\boldsymbol{d}}
$$

And the time derivative of $\boldsymbol{e}$ is

$$
\dot{\boldsymbol{e}}=\dot{\boldsymbol{q}}-\dot{\boldsymbol{q}}_{\boldsymbol{d}}
$$

Finally, the motion dynamics equation of the system becomes:

$$
Q_{C}+\boldsymbol{k}_{D} \dot{\boldsymbol{e}}+\boldsymbol{k}_{P} \boldsymbol{e}+\boldsymbol{k}_{I} \int_{0}^{t} \boldsymbol{e} d t=D(\boldsymbol{q}) \ddot{\boldsymbol{q}}+H(\boldsymbol{q}, \dot{\boldsymbol{q}})+\boldsymbol{G}(\boldsymbol{q})
$$

PID control algorithm expressed as:

$$
Q=\boldsymbol{k}_{P} \boldsymbol{e}+\boldsymbol{k}_{\boldsymbol{I}} \int_{0}^{t} \boldsymbol{e} d t+\boldsymbol{k}_{\boldsymbol{D}} \dot{\boldsymbol{e}}
$$

The control law for a proportional control $(P)$ is:

$$
Q=\boldsymbol{k}_{\boldsymbol{P}} \boldsymbol{e}+Q_{d}
$$

Where $Q_{d}$ is the desired control command and $\boldsymbol{e}$ is an error. The control law for a proportional integral control $(P I)$ is:

$$
Q=\boldsymbol{k}_{P} \boldsymbol{e}+\boldsymbol{k}_{\boldsymbol{I}} \int_{0}^{t} \boldsymbol{e} d t
$$

An integral controller is usually used with a proportional controller. The integral controller usually 
reduces steady state error and help the system to reach the set point. The control law for a proportional derivative control $(P D)$ is:

$$
Q=\boldsymbol{k}_{P} \boldsymbol{e}+\boldsymbol{k}_{D} \dot{\boldsymbol{e}}
$$

A derivative controller is usually used with a proportional controller too. The function of the derivative controller is to make the system more stable with minimum oscillation.

\section{Cart-inverted pendulum with PID}

The cart inveted pendulum has two degrees of freedom $\theta$ and $x$, that selected to be the elements of generalised coordinate vector $\boldsymbol{q}$. So, from the system, we know that:

$$
\begin{gathered}
\boldsymbol{q}=[x, \theta] \\
\boldsymbol{q}_{\boldsymbol{d}}=\left[x_{d}, \theta_{d}\right]
\end{gathered}
$$

The error from the cart inverted described as:

$$
\begin{gathered}
\boldsymbol{e}=\left[\begin{array}{c}
x \\
\theta
\end{array}\right]-\left[\begin{array}{l}
x_{d} \\
\theta_{d}
\end{array}\right] \\
\dot{\boldsymbol{e}}=\left[\begin{array}{c}
\dot{x} \\
\dot{\theta}
\end{array}\right]-\left[\begin{array}{c}
\dot{x}_{d} \\
\dot{\theta}_{d}
\end{array}\right]
\end{gathered}
$$

The proportional gain controller $\boldsymbol{k}_{\boldsymbol{P}}$, derivative gain controller $\boldsymbol{k}_{\boldsymbol{D}}$, and integral gain controller $\boldsymbol{k}_{\boldsymbol{I}}$, described as:

$$
\begin{aligned}
\boldsymbol{k}_{\boldsymbol{P}} & =\left[\begin{array}{ll}
k_{P_{x}} & k_{P_{\theta}}
\end{array}\right] \\
\boldsymbol{k}_{\boldsymbol{D}} & =\left[\begin{array}{ll}
k_{D_{x}} & k_{D_{\theta}}
\end{array}\right] \\
\boldsymbol{k}_{\boldsymbol{I}} & =\left[\begin{array}{ll}
k_{I_{x}} & k_{I_{\theta}}
\end{array}\right]
\end{aligned}
$$

\subsection{The cart of cart inverted pendulum system}

From Eq.(26), the equations for a cart is modified as follow:

$$
\begin{gathered}
Q_{x}=(M+m) \ddot{x}+m l \ddot{\theta} \\
Q_{x_{d}}=(M+m) \ddot{x}_{d}+m l \ddot{\theta}_{d}
\end{gathered}
$$

Eq.(44) is the equation of motion from cart inverted pendulum and Eq.(45) is the equation of force that cart needed for reaching the set point. The control command for cart inverted pendulum is described as follow:

$$
Q_{x}=Q_{x_{d}}-\boldsymbol{k}_{\boldsymbol{P}} \boldsymbol{e}-\boldsymbol{k}_{\boldsymbol{D}} \dot{\boldsymbol{e}}-\boldsymbol{k}_{\boldsymbol{I}} \int_{0}^{t} \boldsymbol{e} d t
$$

The second differential equation of the desired position expressed as

$$
\ddot{x}_{d}=0
$$

$$
\ddot{\theta}_{d}=0
$$

From Eq.(47) and Eq.(48) we know that $\ddot{x}_{d}=0$ and $\ddot{\theta}_{d}=0$, so the equation became

$$
Q_{x}=-\boldsymbol{k}_{\boldsymbol{P}} \boldsymbol{e}-\boldsymbol{k}_{\boldsymbol{D}} \dot{\boldsymbol{e}}-\boldsymbol{k}_{\boldsymbol{I}} \int_{0}^{t} \boldsymbol{e} d t
$$

Substituting Eq.(44) to Eq.(49) the equation became as follow

$$
(M+m) \ddot{x}+m l \ddot{\theta}=-\boldsymbol{k}_{\boldsymbol{P}} \boldsymbol{e}-\boldsymbol{k}_{\boldsymbol{D}} \dot{\boldsymbol{e}}-\boldsymbol{k}_{\boldsymbol{I}} \int_{0}^{t} \boldsymbol{e} d t
$$

The second differential equation of cart position can be expressed as follows

$$
\ddot{x}=\frac{-\boldsymbol{k}_{\boldsymbol{P}} \boldsymbol{e}-\boldsymbol{k}_{\boldsymbol{D}} \dot{\boldsymbol{e}}-\boldsymbol{k}_{\boldsymbol{I}} \int_{0}^{t} \boldsymbol{e} d t-m l \ddot{\theta}}{M+m}
$$

At this point, the differential equation of the cart of cart inverted pendulum motion has been developed.

\subsection{The pendulum of cart inverted pendulum system}

From Eq.(27), the equations for a cart is modified as follow:

$$
\begin{gathered}
Q_{\theta}=\ddot{x}+l \ddot{\theta}-g \theta \\
Q_{\theta_{d}}=\ddot{x}_{d}+l \ddot{\theta}_{d}-g \theta_{d}
\end{gathered}
$$

Eq.(52) is the equation of motion from inverted pendulum and Eq.(53) is the equation of force that pendulum needed for reaching the set point. The control command for pendulum derived as follow:

$$
Q_{\theta}=Q_{\theta_{d}}-\boldsymbol{k}_{\boldsymbol{P}} \boldsymbol{e}-\boldsymbol{k}_{\boldsymbol{D}} \dot{\boldsymbol{e}}-\boldsymbol{k}_{\boldsymbol{I}} \int_{0}^{t} \boldsymbol{e} d t
$$

The second differential equation of the desired position expressed as

$$
\begin{aligned}
& \ddot{x}_{d}=0 \\
& \ddot{\theta}_{d}=0 \\
& \theta_{d}=0
\end{aligned}
$$

From Eq.(55), Eq.(56) and Eq.(57) we know that $\ddot{x}_{d}=0, \ddot{\theta}_{d}=0$, and $\theta_{d}=0$, so the equation became

$$
Q_{\theta}=-\boldsymbol{k}_{\boldsymbol{P}} \boldsymbol{e}-\boldsymbol{k}_{\boldsymbol{D}} \dot{\boldsymbol{e}}-\boldsymbol{k}_{\boldsymbol{I}} \int_{0}^{t} \boldsymbol{e} d t
$$

Substituting Eq.(52) to Eq.(58) the equation became as follow

$$
\ddot{x}+l \ddot{\theta}-g \theta=-\boldsymbol{k}_{\boldsymbol{P}} \boldsymbol{e}-\boldsymbol{k}_{\boldsymbol{D}} \dot{\boldsymbol{e}}-\boldsymbol{k}_{\boldsymbol{I}} \int_{0}^{t} \boldsymbol{e} d t
$$


The second differential equation of degree pendulum expressed as

$$
\ddot{\theta}=\frac{-\boldsymbol{k}_{\boldsymbol{P}} \boldsymbol{e}-\boldsymbol{k}_{\boldsymbol{D}} \dot{\boldsymbol{e}}-\boldsymbol{k}_{\boldsymbol{I}} \int_{0}^{t} \boldsymbol{e} d t-\ddot{x}-g \theta}{l}
$$

At this point, the differential equation of the pendulum of cart inverted pendulum motion have been developed.

\section{Simulation and result}

In order to simulate the linear control technique PID, the cart inverted pendulum parameters defined in Table 1.

Table 1. The Cart Inverted Pendulum System Parameter.

\begin{tabular}{|c|c|c|c|}
\hline No & Parameter & Value & Units \\
\hline 1 & $\mathrm{M}$ & 1 & $\mathrm{Kg}$ \\
\hline 2 & $\mathrm{M}$ & 0.1 & $\mathrm{Kg}$ \\
\hline 3 & 1 & 0.25 & $\mathrm{~m}$ \\
\hline
\end{tabular}

The simulation is done by using the differential equation of pendulum and cart from cart inverted pendulum system which has been obtained. The simulation is done by augmented PID control scheme. The selection of controller parameters $k_{P}, k_{D}$ and $k_{I}$ are by trial and error. Finally, the best response from cart inverted pendulum system has been obtained with the value of $k_{P_{x}} 190, k_{D_{X}} 50, k_{I_{X}} 5, k_{P_{\theta}} 140, k_{D_{\theta}} 5$, and $k_{I_{\theta}} 25$. It can be seen in Fig. 4 that the best response are shown by the cart inverted pendulum system with the pendulum response reaches the desired position within 2 seconds and the cart response reaches the desired position in less than 4 seconds.
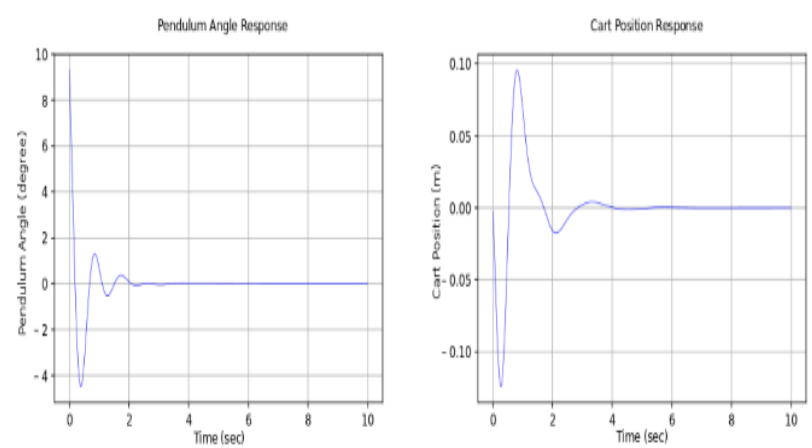

Fig. 4. Best pendulum angle and cart response trajectories.

Furthermore, the simulation is done by changing the parameter of the PID controller. This is done to determine the pendulum and cart response to the system in case of PID parameter change. The first simulation is done by changing $k_{P_{x}} \cdot k_{P_{x}} 20$ and 50 applied to the simulation. From Fig.5, it can be concluded that the greater $k_{P_{X}}$ make the system responses can achieve the desired position quickly. If $k_{P_{X}}$ is too small, the system becomes unstable.
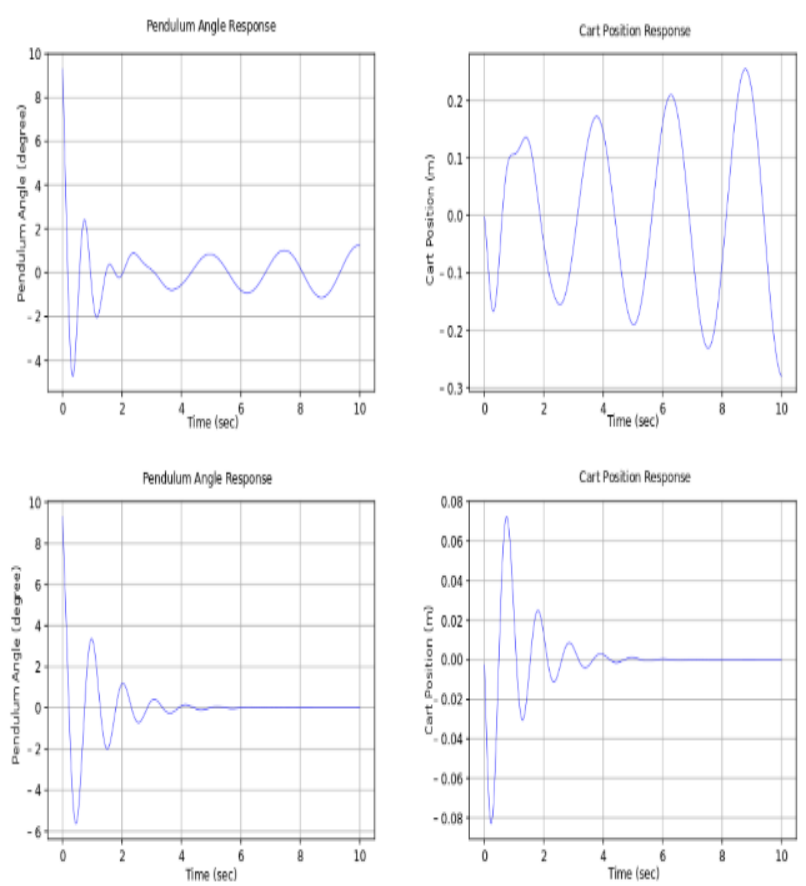

Fig. 5. Pendulum angle and cart response trajectories with variation of $\boldsymbol{k}_{\boldsymbol{P}_{\boldsymbol{X}}}$.

$k_{D_{x}}$ changes are made with $k_{D_{x}} 10$ and $k_{D_{x}} 140$. The results from simulations are shown in Fig. 6. It is known that small $k_{D_{x}}$ can stabilize the system, but higher $k_{D_{x}}$ can make the system unstable.
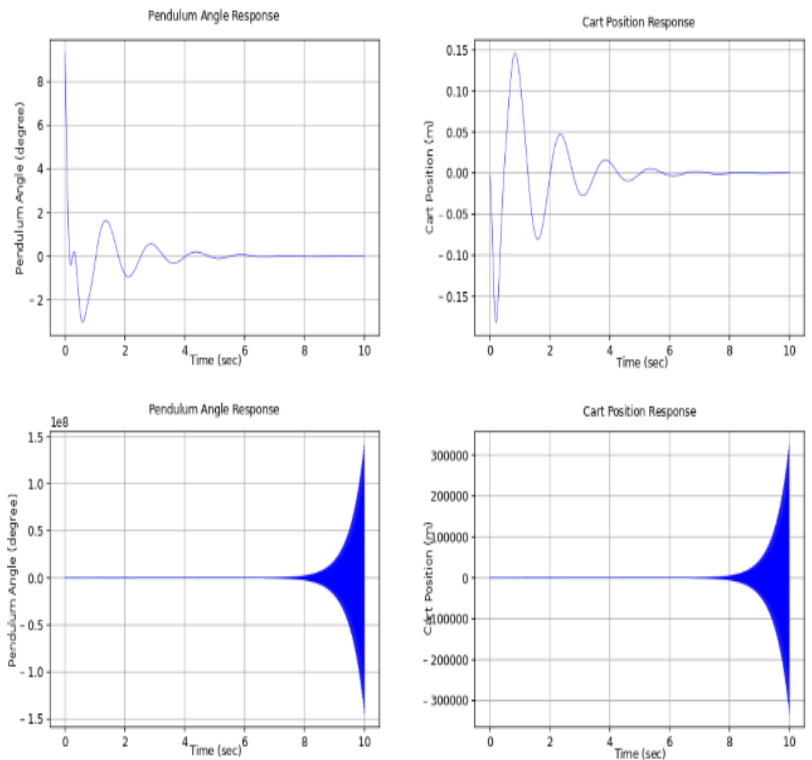

Fig. 6 Pendulum angle and cart response trajectories with variation of $\boldsymbol{k}_{\boldsymbol{D}_{\boldsymbol{x}}}$.

The next step is change the value of $k_{I_{x}}$. The small $k_{I_{x}}$ does not affect system response at all. But if the $k_{I x}$ that given to the system is too large, makes the system unstable. Fig.7 shows the pendulum and cart response on a cart inverted pendulum system with a value of $k_{I_{X}} 0$ and $k_{I_{x}} 50$. 

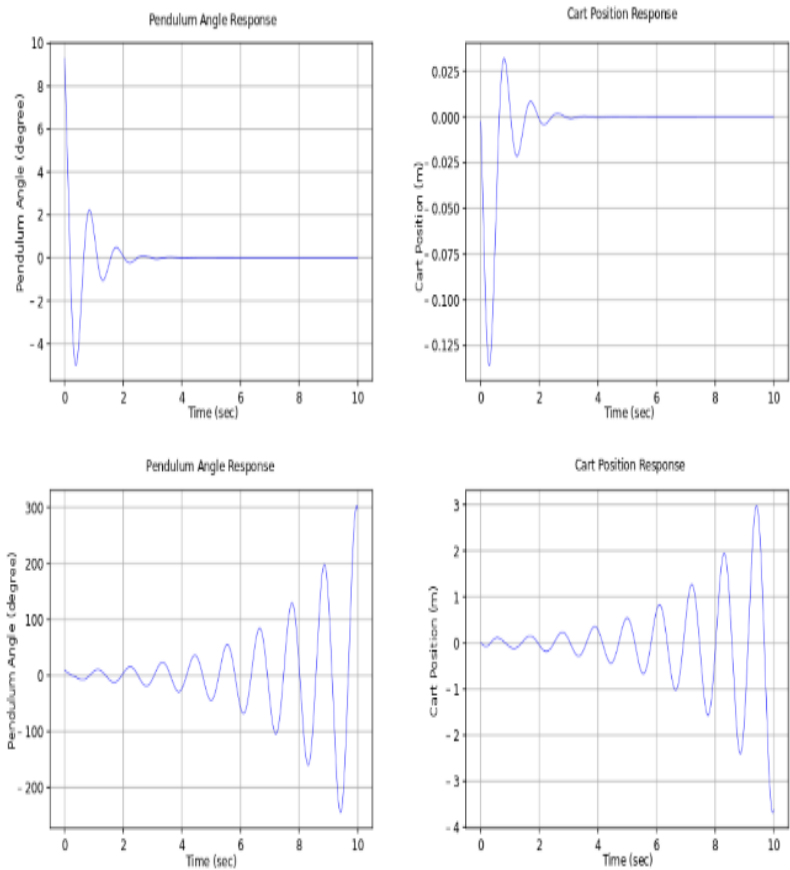

Fig. 7. Pendulum angle and cart response trajectories with variation of $k_{I_{x}}$.

The next simulation is replacing the parameter PID on $\theta$. The first thing to do is replacing the $k_{P_{\theta}}$. Fig. 8 shows the $k_{P_{\theta}} 10$ and 600 . From Fig. 8, it can be seen that the change in $k_{P_{\theta}}$ does not significantly affect the system response.
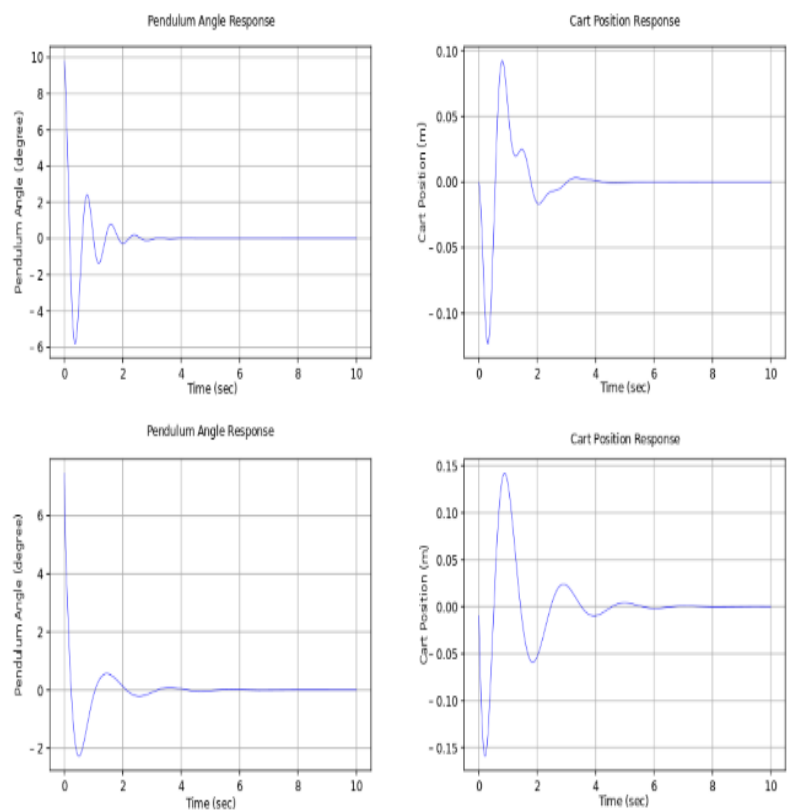

Fig. 8. Pendulum angle and cart response trajectories with variation of $\boldsymbol{k}_{\boldsymbol{P} \boldsymbol{\theta}}$.

The $k_{D_{\theta}}$ changes are done with 0 and 20 . It can be seen in Fig.9 that the small $k_{D_{\theta}}$ does not affect the system response. But if the given $k_{D_{\theta}}$ is slightly higher, the system becomes unstable.
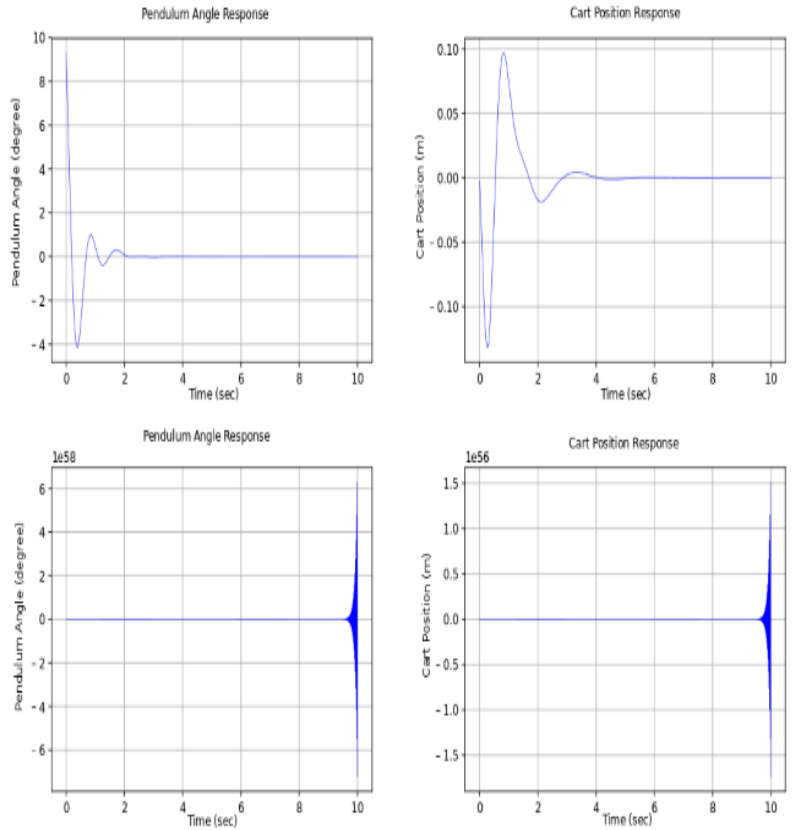

Fig. 9. Pendulum angle and cart response trajectories with variation of $\boldsymbol{k}_{\boldsymbol{D}_{\boldsymbol{\theta}}}$.

The last one is the change of $k_{I_{\theta}}$ with the gain 5 and 50. From Fig. 10, it can be seen that the higher value of the $k_{I_{\theta}}$ makes the system reach the desired point faster.
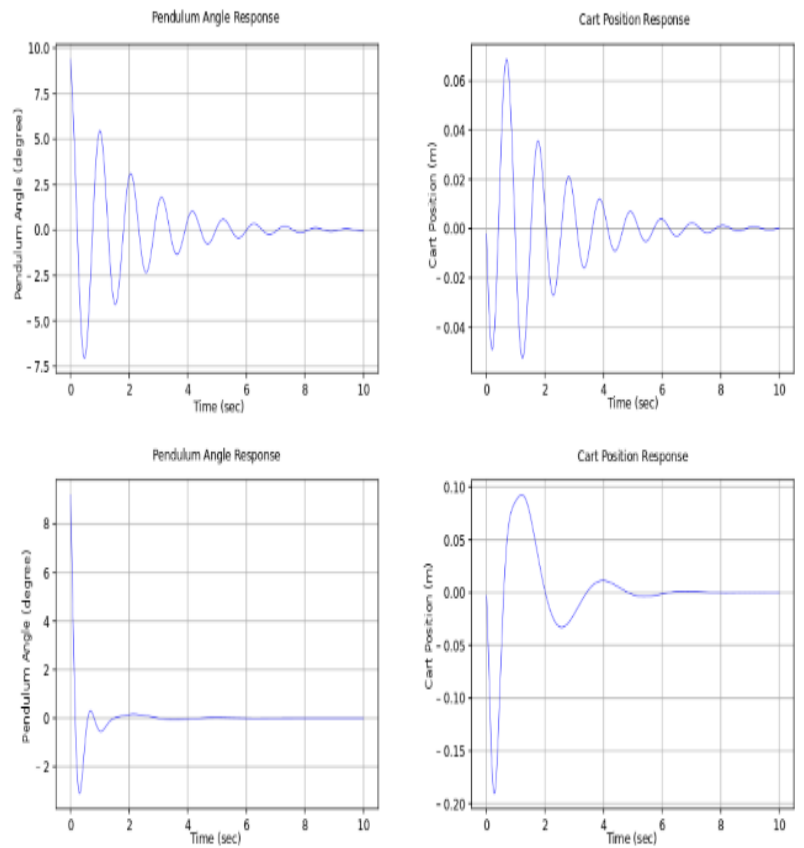

Fig. 10. Pendulum angle and cart response trajectories with variation of $\boldsymbol{k}_{\boldsymbol{I}_{\boldsymbol{\theta}}}$.

It can be concluded that the selection of PID parameters on $k_{P_{x}}, k_{D_{x}}, k_{I_{x}}, k_{P_{\theta}}, k_{D_{\theta}}$, and $k_{I_{\theta}}$ greatly affects the system response.

\section{Conclusions}

The mathematical model of a cart inverted pendulum has been successfully conducted using Lagrange equation. 
The control command for cart and pendulum in a cart inverted pendulum successfully made the system reach the desired point. Linear control technique is a simple control technique based on the linearisations of the equation of motion. The parameter of proportional gain $\boldsymbol{k}_{\boldsymbol{P}}$, the integral gain $\boldsymbol{k}_{\boldsymbol{I}}$, and derivative gain $\boldsymbol{k}_{\boldsymbol{D}}$ have been successfully selected to satisfy the system stability conditions.

\section{References}

1. L. B. Prasad, B. Tyagi, and H. O. Gupta, Optimal control of nonlinear inverted pendulum system using pid controller and lqr: Performance analysis without and with disturbance input, Int. J. of Automation and Computing, vol. 11, no. 6, pp. 661670, (2014)

2. G. V. Troshina, A. A. Voevoda, and K. M. Bobobekov, The parameters determination of the inverted pendulum model in the automatic control system, in 2016 13th International Scientific Technical Conference on Actual Problems of Electronics Instrument Engineering (APEIE), vol. 03 pp. 180-182 (2016)

3. K. Shibayama, V. Kroumov, and A. Inoue, Robust control of underactuated inverted pendulum system in presence of unknown disturbances, in Proceedings of the 2010 International Conference on Modelling, Identification and Control, July, pp. 349-353 (2010)

4. I. Siradjuddin, Z. Amalia, B. Setiawan, R. P. Wicaksono and E. Yudaningtyas, Stabilising a cart inverted pendulum system using pole placement control method, 2017 15th International Conference on Quality in Research (QiR) : International Symposium on Electrical and Computer Engineering, Nusa Dua, pp. 197-203 (2017)

5. N. S. Reddy, M. S. Saketh, P. Pal and R. Dey, Optimal PID controller design of an inverted pendulum dynamics: A hybrid pole-placement and firefly algorithm approach, 2016 IEEE First International Conference on Control, Measurement and Instrumentation (CMI), Kolkata, pp. 305-310 (2016)

6. R. Oróstica, M. A. Duarte-Mermoud and C. Jáuregui, Stabilization of inverted pendulum using LQR, PID and fractional order PID controllers: A simulated study, 2016 IEEE International Conference on Automatica (ICA-ACCA), Curico, pp. 1-7 (2016)

7. S. D. Hanwate, A. Budhraja and Y. V. Hote, Improved performance of cart inverted pendulum system using LQR based PID controller and ANN, 2015 IEEE UP Section Conference on Electrical Computer and Electronics (UPCON), Allahabad, pp. 1-6 (2015)

8. V. R. Molazadeh, A. Banazadeh, and I. Shafieenejad, Design of the lqr controller and observer with fuzzy logic ga and ga-pso algorithm for triple an inverted pendulum and cart system, in Proceedings of the 2014 International Conference on Advanced Mechatronic Systems, pp. 295-300 (2014)

9. R. Sharma, Game theoretic lyapunov fuzzy control for inverted pendulum, in 2015 4th International Conference on Reliability, Infocom Tech- nologies and Optimization (ICRITO) (Trends and Future Directions), pp. 1-6 (2015)

10. M. Salem and M. F. Khelfi, Nonlinear inverted pendulum pid control by an improved artificial bees colony-predator and prey approach, in $3 r d$ International Conference on Systems and Control, pp. 792-796 (2013) 